\title{
Sensory impairments and health concerns related to the degree of intellectual disability in people with Down syndrome
}

\author{
Tuomo Määttä ${ }^{1}$, Markus Kaski ${ }^{2}$, Anja Taanila ${ }^{3}$, Sirkka Keinänen- \\ Kiukaanniemi ${ }^{3}$ and Matti livanainen ${ }^{2,4}$
}

\begin{abstract}
${ }^{1}$ Service Centre of Kuusanmäki, ${ }^{2}$ Rinnekoti Research Centre, ${ }^{3}$ Department of Public Health Science and General Practice, University of Oulu, and ${ }^{4}$ Department of Child Neurology, University of Helsinki, Finland
\end{abstract}

\begin{abstract}
The relationship between poor health and cognitive impairment is not fully understood yet. People with Down syndrome are prone to a number of health problems, including congenital heart defect, visual impairment, hearing loss, autoimmune diseases, epilepsy, early-onset Alzheimer's disease and intellectual disability. Our aim was to assess the impact of impaired health on cognitive performance in people with Down syndrome. A series of people with Down syndrome $(n=129)$ were studied for their intellectual disability, sensory impairments and health concerns. The medical and psychological records of all persons with Down syndrome in the Intellectual Disability Service Register of Kainuu from 1970 to 2004 were analysed. The detected health issues were related to the individuals' cognitive levels. Visual impairment, poor dental health and acute neurological disease were associated with the severity of intellectual disability and ageing.
\end{abstract}

Keywords: Down syndrome, intellectual disability, sensory impairments, visual impairment, hearing loss, health situation, stroke, dental health

\section{Introduction}

Any disease affecting brain function may affect learning and behaviour. Intellectual disability may be caused by any condition seriously affecting the development and function of the central nervous system during intrauterine and later life up to 18 years of age. Short-term memory functions have been identified as predictors of language and comprehension skills in children with Down syndrome (Chapman \& Hesketh, 2001). The level of intellectual disability is not necessarily a permanent condition but may change downwards or upwards during development and ageing. Learning and acquisition of skills in childhood and adolescence may be followed by a loss of abilities in later life of persons with Down syndrome (Prasher \& Chung, 1996).

People with Down syndrome have an increased risk of visual and hearing impairment compared to individuals with typical development or intellectual disabilities due to other aetiologies (Merrick \& Koslowe, 2001; Shott et al., 2001; van Splunder et al. 2003; Roizen \& Patterson, 2003). In general, visual impairments are more frequent 
T. Määttä et al. • Sensory impairments and health concerns related to the degree of intellectual disability

in people with severe and profound intellectual disability than in persons with mild or moderate intellectual disability (van Schrojenstein Lantman-de Valk et al., 1994; Evenhuis et al., 2001).

Good hearing ability during early childhood is important for language (Wake et al., 2004) and cognitive development (Davis \& Hind, 1999). Early intervention with hearing aids and family involvement correlate with positive language outcomes in children with congenital hearing loss (Moeller, 2000). Early diagnosis and treatment of illnesses, e.g., middle ear infections, causing hearing impairment in children with Down syndrome provides significantly improved hearing levels compared to delayed diagnosis and treatment (Shott et al., 2001).

People with Down syndrome have an increased incidence of congenital heart defects, thyroid disease, coeliac disease, diabetes, depression and Alzheimer's disease (Roizen \& Patterson, 2003). Other risks to cognitive abilities include central nervous system infections, stroke and accidents. All these diseases may affect cognitive functions by interfering with circulation, nutrition or metabolism of the brain.

Nowadays, people with Down syndrome live longer than before and therefore become prone to health problems associated with ageing (Yang et al., 2002). Ageing, dementia and the severity of intellectual disability are significant factors leading to decline in adaptive behaviour over advancing age in adults with Down syndrome (Prasher \& Chung, 1996). Thus, absence of a medical illness predicts a higher level of adaptive behaviour. There is a need for better understanding of the health concerns of this population (Merrick \& Kandel, 2003). The development of comprehensive medical services is essential to ensure a good quality of life for persons with Down syndrome (Smith, 2001; Roizen \& Patterson, 2003).

The aim of this study was to assess the impact of impaired health on cognitive performance in people with Down syndrome.

\section{Material and methods}

A population-based series of people with Down syndrome in northern Finland were studied for their sensory impairments and other health concerns. Case records of all persons with Down syndrome $(n=129)$ in the Intellectual Disability Service Register of Kainuu were identified and analysed. The medical records of individuals $(n=116)$ in the central hospital providing secondary specialised physical and mental health care in this region were surveyed by the first author for any clinically significant health problems. able 1. Characteristics of the study population $(n=129)$

\begin{tabular}{lccc} 
& & Males $(\mathrm{n}=\mathbf{7 6})$ & Females $(\mathrm{n}=\mathbf{5 3})$ \\
\hline Birth year & range & $1933-2002$ & $1933-2003$ \\
Age at last visit (years) & range & $0-60.8$ & $0-66.7$ \\
& mean & 29 & 35 \\
Medical records $(\mathrm{g})$ & range & $0-2130$ & $0-1340$ \\
& mean & 489 & 494
\end{tabular}

The ethics committee of Kainuu Hospital District approved the study. The Finnish Ministry of Social Affairs and Health approved the linkage of social and health register data needed in the study.

Data was collected from repeated medical and psychological evaluations from January 1970 till March 2004, totalling 4057 person-years. The time of the last evaluation varied between the years 1975 and 2004, and the age at the last evaluation varied between 0.1 and 66.7 (mean 31.4 , standard deviation 16.8 years) years. There were 76 (59\%) male and 53 (41\%) female participants (Table 1).

The detected health concerns were summarised according to the diagnostic criteria of the International Classification of Diseases and Related Health Problems (ICD-10, World Health Organization, 1992). The ICD-10 criteria for intellectual disability include adaptive behaviour in addition to cognitive abilities. Scoring of health topics and the degree of intellectual abilities was used to explore and describe possible correlations between health and cognitive outcome.

Intellectual disability had been determined by repeated psychological assessments. The severity of intellectual disability was scored from 1 to 4 according to the best level achieved by each individual as follows: mild (IQ 5069) 1; moderate (IQ 35-49) 2; severe (IQ 20-34) 3; profound intellectual disability (IQ < 20) 4.

Speech production was used as an index of verbal communication abilities. Speech impairment was scored as follows: relatively fluent speech with extensive vocabulary 0 ; communicative speech with long sentences 1 ; short sentences 2; single words 3; no verbal communication 4 .

Visual impairment was scored as follows: visual acuity of the better eye with or without correction of refraction at least 0.80 ; visual acuity $0.4-0.71$; visual acuity not known 2 ; visual acuity $0.1-0.3$ or cataracts or corneal opacities 3; visual acuity less than 0.14 ; bilateral blindness 5.

Hearing loss and ear disease were scored as follows: hearing thresholds at frequencies of $0.5-1 \mathrm{kHz}$ in the better ear less than $20 \mathrm{~dB}$ in audiometry 0 ; hearing thresholds less than $30 \mathrm{~dB}$ by screening audiometry at $1.0 \mathrm{~Hz} \mathrm{1}$; 
hearing not determined or evaluated as normal 2; hearing thresholds 20-49 $\mathrm{dB}$ in audiometry or less than $50 \mathrm{~dB}$ by screening audiometry, recurrent middle ear infections, glue ear or perforation of tympanic membrane 3 ; hearing thresholds 50 $70 \mathrm{~dB}$ in audiometry or less than $70 \mathrm{~dB}$ by screening audiometry or hearing aids needed 4; hearing thresholds more than $70 \mathrm{~dB} 5$. Pocket Audiometry Tester (PAT225 of Madsen electronics) was used for screening audiometry.

Dental health scoring was based on the number of lost permanent teeth as follows: no loss of permanent teeth 0 ; loss of 1-4 permanent teeth 1 ; loss of 5-9 permanent teeth or the number of lost teeth unknown 2; loss of 10 or more permanent teeth with more than 5 remaining teeth, or repeated treatments under general anaesthesia needed 3; only 15 permanent teeth left or severe caries or chronic periodontal infection 4 ; loss of all permanent teeth 5 .

Immune diseases were scored according to the number of autoimmune and related diseases, including atopic dermatitis, asthma, coeliac disease, thyroid disease, psoriasis, alopecia, rheumatoid arthritis and diabetes.

Infectious diseases other than dental or upper respiratory infections or ear infections were scored as follows: no episodes of pneumonia, urinary tract infections, abdominal or skin infections 0 ; one or two episodes 1 ; three or more episodes, chronic infection or very serious (septic) infections 2.

Epilepsy and stroke score was defined as the sum of the following items: recurrent fainting 1; epilepsy 1 ; number of episodes of stroke 0-5; hemiplegia 1; bilateral involvement 2; visual impairment 1 , and speech loss 1 . The score was used as an index of neurological symptoms (including fainting, epilepsy and stroke) possibly or definitely related to impairment of brain circulation.

Behavioural and mental health problems were separately scored for severity as follows. Mood, anxiety: no problems 0; mild depression/withdrawal 1; moderate depression, anxiety or fears 2; severe depression, anxiety, somatic symptoms 3; very severe depression, psychotic symptoms or severe self-injury 4 . Behaviour: no problems 0; occasional difficulties 1; occasional aggressive outbursts, destruction of material objects 2 ; severe irritability, disturbing behaviour, attacking others 3; difficult to manage, dangerous to others 4 .

The data was analysed using the Microsoft Excel and SPSS 11.5 for Windows software packages. Descriptive statistics and non-parametric correlation (Spearman) for statistical significance were used. Partial correlations were used to control the effects of age and birth year.
Table 2. Intellectual disability by age $(n=121)$

\begin{tabular}{lcccccc} 
& \multicolumn{2}{c}{$0-19$} & \multicolumn{2}{c}{$20-39$} & \multicolumn{2}{c}{$40-$} \\
& count & $\%$ & count & $\%$ & count & $\%$ \\
mild & 16 & 42 & 5 & 12 & 2 & 5 \\
moderate & 11 & 29 & 13 & 32 & 12 & 28 \\
severe & 7 & 18 & 15 & 37 & 18 & 43 \\
profound & 4 & 11 & 8 & 19 & 10 & 24 \\
\hline
\end{tabular}

i-Square Tests

Pearson Chi-Square 22.065, df 6, Asymp. Sig. (2-sided) $p<.001$

kelihood Ratio 21.906, df 6, Asymp. Sig. (2-sided) $p<.001$

inear-by-Linear Association 15.292, df 1, Asymp. Sig. (2-sided) $p<.000$

\section{Results}

The cognitive abilities of 121 individuals with Down syndrome out of 129 had been evaluated. The degree of intellectual disability was mild in 23 (19\%), moderate in $36(30 \%)$, severe in $40(33 \%)$ and profound in $22(18 \%)$ persons. Older age groups had more severe intellectual disability compared to younger groups (Table 2).

The speech production of the 115 persons evaluated consisted of at least short sentences in $50(43 \%)$ cases, single words in 40 (35\%) and no speech in 25 (22\%).

The sensory and health concerns of 129 persons included visual impairment with visual acuity of 0.3 or less in 30 individuals (23\%), hearing loss with hearing thresholds of $20 \mathrm{~dB}$ or more in audiometry or recurrent middle ear infections in $42(33 \%)$, loss of at least 10 permanent teeth, severe caries or periodontal disease in $47(36 \%)$, at least one immune disease in 47 (36\%), at least one episode of infection (excluding upper respiratory and ear infections) treated in hospital in $56(43 \%)$ and stroke or suspected stroke in $14(11 \%)$.

The weight of the hard-copy medical records varied between 0 and 2130 grams (mean $493 \mathrm{~g}$, SD $393 \mathrm{~g}$ ) and correlated with the frequency of infections (0.42), the severity of heart defect related symptoms (0.39), the year of birth (0.32), the stroke score (0.24) and the presence of immune disease $(0.17)$, but the correlation with intellectual disability was negative (-0.29).

The correlations between intellectual disability, speech impairment and age with visual and hearing impairments, dental loss, epilepsy and stroke are presented in Table 3.

The severity of intellectual disability was closely related to the difficulty of verbal communication. Higher levels of cognitive abilities correlated positively with better speech. The severity of intellectual disability had a significant positive correlation with increasing age, dental loss and visual impairment at the 0.01 level and with 
T. Määttä et al. • Sensory impairments and health concerns related to the degree of intellectual disability

the epilepsy and stroke score at the 0.05 level (Table 3.). We also found a significant positive correlation $(p<0.05)$ of hearing impairment (including ear disease) with infections in general, poor speech production and young age.

Advanced age at the last visit had a significant positive correlation with the severity of intellectual disability, dental loss, the epilepsy and stroke score $(p<0.01)$ and visual impairment $(p<0.05)$, but the correlation between age and hearing loss was negative $(p<0.01) \quad$ (Table 3.).

After adjustment for age and birth year, partial correlation coefficients showed a significant correlation between the severity of intellectual disability and speech impairment, visual impairment, dental loss $(p<0.01)$ and hearing impairment $(p<0.05)$. Speech impairment had a significant positive correlation with hearing impairment $(p<0.01)$, visual impairment, dental loss and the epilepsy and stroke score $(p<0.05)$ (Table 4.)

\section{Discussion}

The severity of intellectual disability correlated with the difficulty of verbal communication. Higher levels of cognitive abilities correlated with better speech. Advancing age was associated with more severe intellectual disability. Most probably, learning difficulties resulted in relatively slow acquisition of language and other skills during childhood. The degree of intellectual disability was sometimes estimated only during adulthood, when dementia and ageing may already have affected the person's abilities.

The observation that visual impairment was more common in people with severe to profound intellectual disability than in those with mild to moderate intellectual disability confirms the findings reported earlier (van Schrojenstein Lantman-de Valk et al., 1994, Evenhuis et al., 2001) and suggests the contribution of poor vision to cognitive impairment. Van Splunder et al. (2003) found a significant correlation of progressive amounts of strabismus and ocular pathology with increasing learning difficulty in children with Down syndrome, but no correlation was found between refractive errors and the level of learning difficulty. Regular screening of vision followed by proper use of glasses when needed is helpful. Use of bifocal glasses to correct the common accommodation deficit in children with Down syndrome (Cregg et al., 2001) has been recommended.

Our study detected an association between hearing impairment and the severity of intellectual disability when the effects of age were controlled for by using partial correlation coefficients. The most obvious explanation is that hearing loss was manifested at young age. Other studies have also provided only limited evidence of hearing loss affecting cognitive functions in people with Down syndrome. Libb et al. (1985), for instance, reported that children and young adults with Down syndrome and abnormal tympanograms performed less well in intelligence tests compared to those with normal tympanograms. In people with Down syndrome, the correlation of hearing variables with seven cognitive tasks administered on the same day revealed only one significant relationship after the statistical removal of the effects of cognitive age and intelligence quotient: subjects with poorer hearing identified fewer words in a task in which a masking noise quickly followed a spoken word (Marcell \& Cohen, 1992). This surprising observation

\begin{tabular}{lcc}
\hline $\begin{array}{l}\text { Table 4. Partial correlation coefficients } \\
\text { at last visit and birth year) }\end{array}$ & Intellectual disability & Speech impairment \\
\hline Intellectual disability & 1.000 & $\left..702^{(* *}\right)$ \\
Speech impairment & $\left..702^{* *}\right)$ & 1.000 \\
Visual impairment & $\left..328^{* *}\right)$ & $\left..191^{*}\right)$ \\
Hearing impairment & $\left..171^{*}\right)$ & $.27\left(^{* *}\right)$ \\
Dental loss & $\left..252^{* *}\right)$ & $\left..197^{*}\right)$ \\
Epilepsy \& stroke & .094 & $.208\left(^{*}\right)$ \\
\hline
\end{tabular}

** Correlation is significant at the 0.01 level (1-tailed).

* Correlation is significant at the 0.05 level (1-tailed). 
might be partially explained by the methods used in the psychometric evaluations, which was obviously based more on visual acuity than on hearing. The difficulty of detecting hearing impairment in people with Down syndrome is another explanation. The tendency for sensory impairments to go unnoticed in people with intellectual disabilities is considerable (Evenhuis et al. 2001). Probably not all persons with hearing impairment could be identified. A reliable evaluation of hearing had been performed and recorded for a minority of people in this sample. One could expect, however, that any clinically severe hearing impairment detected would have been evaluated and recorded in the specialised health care records surveyed.

The treatment of middle ear infections and glue ear has been active in Finland, and this could be one reason for the low frequency of detected hearing impairment in the present study. Aggressive treatment of middle ear disease in children with Down syndrome started soon after birth provides significantly better hearing levels (Shott et al., 2001) than previously reported.

Short-term memory impairment is a common finding in people with Down syndrome, and it has even been regarded as an aspect of the behavioural phenotype of Down syndrome (Chapman \& Hesketh, 2001). The impairment of auditory short-term memory is thought to contribute to difficulties of language acquisition, speech and cognition even more than hearing impairment (Jarrold et al., 2002).

The association between dental health and intellectual abilities has many possible explanations. Dental loss was associated with greater age and, hence, with a more severe degree of intellectual disability. Adults with intellectual disability and poor ability to co-operate with dental treatment and those with Down syndrome have an increased risk for impaired oral health (Gabre et al, 2001).

No published population-based epidemiological studies of stroke in people with Down syndrome are available so far. The fact that stroke was common in the present study population, especially in those with poor cognitive outcome, suggests that stroke probably contributes significantly to cognitive impairment in individuals with Down syndrome.

In conclusion, this paper deals with the health concerns of persons with Down syndrome at different levels of intellectual disability. Basically, the more severe the degree of intellectual disability in people with Down syndrome, the more severe are their health concerns. This study also confirms the complexity of the health concerns present in this population. Ordinary illnesses are common, and behavioural changes or loss of function may be the only indications of medical illnesses. Nowadays, people with Down syndrome live longer and are therefore becoming prone to age-related health problems and diseases of old age. There is a need to understand better the health needs of this population, to optimise their cognitive and functional performance and to avoid preventable and treatable disabilities.

\section{Acknowledgements}

The study was supported by grants from the Finnish Cultural Foundation and the Rinnekoti Research Foundation.

\section{Correspondence}

Tuomo Määttä • Service Centre of Kuusanmäki, 87250 Kajaani, Finland •tuomo.maatta@kainuu.f. fax: +358 8 61562932 • Tel: +358861562905

\section{References}

Chapman, R.S. \& Hesketh, L.J. (2001). Language, cognition, and short-term memory in individuals with Down syndrome. Down Syndrome Research and Practice, 7(1), 1-7.

Cregg, M., Woodhouse, J.M., Pakeman, V.H., Saunders, K.J., Gunter, H.L., Parker, M., Fraser, W.I. \& Sastry, P. (2001). Accommodation and refractive error in children with Down syndrome: cross-sectional and longitudinal studies. Investigative Ophthalmology and Visual Science, 42(1), 55-63.

Davis, A. \& Hind, S. (1999). The impact of hearing impairment: a global health problem. International Journal of Pediatric Otorhinolaryngology, 5, 49 Suppl 1:S51-4.

Evenhuis, H.M., Theunissen, M., Denkers, I., Verschuure, H. \& Kemme, H. (2001). Prevalence of visual and hearing impairment in a Dutch institutionalised population with intellectual disability. Journal of Intellectual Disability Research, 45(Pt 5), 457-64.

Gabre, P., Martinsson, T. \& Gahnberg, L. (2001). Longitudinal study of dental caries, tooth mortality and interproximal bone loss in adults with intellectual disability. European Journal of Oral Science, 109(1), 20-6.

Jarrold, C., Baddeley, A.D. \& Phillips, C.E. (2002). Verbal short-term memory in Down syndrome: a problem of memory, audition, or speech? Journal of Speech, Language and Hearing Research, 45(3), 53144.

Libb, J.W., Dahle, A., Smith, K., McCollister, F.P. \& McLain C. (1985). Hearing disorder and cognitive function of individuals with Down syndrome. American Journal of Mental Deficiency, 90(3), 353-6. 
T. Määttä et al. • Sensory impairments and health concerns related to the degree of intellectual disability

Merrick, J. \& Koslowe, K. (2001). Refractive errors and visual anomalies in Down syndrome. Down Syndrome Research and Practice, 6(3), 131-3.

Marcell, M.M. \& Cohen, S. (1992). Hearing abilities of Down syndrome and other mentally handicapped adolescents. Research of Developmental Disability, 13(6), 533-51.

Merrick, J. \& Kandel, I. (2003). Health needs of adults with intellectual disability relevant for the family physician. Scientific World Journal, 5(3), 937-45.

Moeller, M.P., (2000). Early intervention and language development in children who are deaf and hard of hearing. Pediatrics, 106(3), E43.

Prasher, V.P. \& Chung, M.C. (1996). Causes of agerelated decline in adaptive behaviour of adults with Down syndrome: differential diagnoses of dementia. American Journal of Mental Retardation, 101(2), 17583.

Roizen, N.J. \& Patterson, D. (2003). Down's syndrome. Lancet, 361(12), 1281-89.

van Schrojenstein Lantman-de Valk, H.M., Haveman, M.J., Maaskant, M.A., Kessels, A.G., Urlings, H.F. \& Sturmans, F. (1994). The need for assessment of sensory functioning in ageing people with mental handicap. Journal of Intellectual Disability Research, 38(Pt 3), 289-98.

Shott, S.R., Joseph, A. \& Heithaus, D. (2001). Hearing loss in children with Down syndrome. International Journal of Pediatric Otorhinolaryngology, 1;61(3), 199 205.

Smith, D.S. (2001). Health care management of adults with Down syndrome. American Family Physician, $15 ; 64(6), 1031-8$

van Splunder, J., Stilma, J.S., Bernsen, R.M., Arentz, T.G., Evenhuis, H.M. (2003). Refractive errors and visual impairment in 900 adults with intellectual disabilities in the Netherlands. Acta Ophthalmologica Scandinavica, 81(2), 123-9.

Wake, M., Hughes, E.K., Poulakis, Z., Collins, C. \& Rickards, F.W. (2004). Outcomes of children with mild-profound congenital hearing loss at 7 to 8 years: a population study. Ear Hear, 25(1), 1-8.

World Health Organization (WHO) (1992). International Classification of Diseases - 10th revision (ICD10). World Health Organization, Geneva.

Yang, Q., Rasmussen, S.A. \& Friedman, J.M. (2002). Mortality associated with Down's syndrome in the USA from 1983-1997: a population-based study. Lancet, 359, 1019-25. 